\title{
Magnetic properties of silica coated spindle-type hematite particles
}

\author{
M Reufer ${ }^{1}$, H Dietsch ${ }^{1}$, U Gasser ${ }^{1,2}$, B Grobety ${ }^{3}$, A M Hirt ${ }^{4}$, \\ V K Malik ${ }^{5}$ and P Schurtenberger ${ }^{1,6}$ \\ ${ }^{1}$ Adolphe Merkle Institute and Fribourg Center for Nanomaterials, University of Fribourg, \\ 1723 Marly, Switzerland \\ ${ }^{2}$ Laboratory for Neutron Scattering, Paul Scherrer Institut, 5232 Villigen PSI, Switzerland \\ ${ }^{3}$ Department of Geosciences and Fribourg Center for Nanomaterials, University of Fribourg, \\ 1700 Fribourg, Switzerland \\ ${ }^{4}$ Institute of Geophysics, ETH Zürich, 8092 Zürich, Switzerland \\ ${ }^{5}$ Department of Physics and Fribourg Center for Nanomaterials, University of Fribourg, \\ 1700 Fribourg, Switzerland \\ ${ }^{6}$ Physical Chemistry, Department of Chemistry, Lund University, S-221 00 Lund, Sweden
}

E-mail: Peter.Schurtenberger@fkem1.lu.se

\begin{abstract}
Magnetic properties of particles are generally determined from randomly oriented ensembles and the influence of the particle orientation on the magnetic response is neglected. Here, we report on the magnetic characterization of anisotropic spindle-type hematite particles. The easy axis of magnetization is within the basal plane of hematite, which is oriented perpendicular to the spindle axis. Two standard synthesis routes are compared and the effects of silica coating and particle orientation on the magnetic properties are investigated. Depending on the synthesis route we find fundamentally different magnetic behavior compatible with either single domain particles or superparamagnetic sub-units. Furthermore, we show that silica coating reduces the mean blocking temperature to nearly room temperature. The mechanical stress induced by the silica coating appears to reduce the magnetic coupling between the sub-units.
\end{abstract}

\section{Introduction}

Anisotropic magnetic particles are receiving considerable attention not only in the traditional field of data storage applications, but more recently as model systems with anisotropic interactions [1, 2]. Moreover, magnetic nanoparticles are key to magnetic (protein) separation techniques [3, 4], and find medical applications in oncology or as magnetic contrast agents [5]. For these applications, detailed knowledge of the magnetic properties on the microscopic scale and the response in an applied magnetic field is essential. It is well known that the micromagnetic structure of magnetic particles is affected by their crystalline structure and their shape through the magnetocrystalline, shape and other magnetic anisotropy energies $[6,7]$. This can lead to a directional dependence of the magnetic properties either due to particle shape for particles dominated by magnetostatic anisotropy, or crystal orientation for particles dominated by magnetocrystalline anisotropy. How this affects the magnetic properties of an ensemble of particles is not well understood. Most of the magnetic measurements on nanoparticles are made with randomly oriented particles [8-10], and hence do not consider anisotropy on the microscopic level. Moreover, when dispersed in a solvent, particles align their magnetization parallel to an applied external magnetic field. In this case the bulk magnetic properties of the system are not relevant but only those in the energetically favorable direction (easy axis).

In this context, spindle-type hematite particles deserve special attention both from a fundamental and a technological point of view. Several authors have studied the magnetic properties of spindle-type hematite particles [8, 11, 12], but only the bulk magnetic properties have been reported. In this study we are not only interested in bulk magnetic properties, but also account for the particle anisotropy. Hematite 
$\left(\alpha-\mathrm{Fe}_{2} \mathrm{O}_{3}\right)$ is the most stable phase of iron oxide in air at ambient conditions. It is only weakly ferromagnetic, so that dipole-dipole interactions do not affect measurements on highly concentrated systems, such as pressed powders. In contrast to most ferrofluids, hematite particles can be readily produced in ellipsoidal shape with variable aspect ratios and small polydispersity [13, 14]. Hematite particles can also be easily coated by a silica layer with controlled thickness $[15,16]$. A silica coating is of particular interest for biocompatibility and for enhanced colloidal stability at neutral $\mathrm{pH}[15,16]$. Moreover, the surface chemical functionality can afterward be engineered via a number of silane coupling agents, facilitating the incorporation of the silica coated hematite particles into any desired environment such as organic solvents or a polymer matrix [17] or polymer shell [18]. This study is novel in that it not only investigates the influence of a silica coating on the magnetic properties of hematite particles, but also considers the anisotropic shape (spindle-type) of the particles.

Hematite has a corundum-type crystal structure with space group $R \overline{3} c$. Above the Morin transition temperature $T_{\mathrm{M}}$ (263 $\mathrm{K}$ in bulk hematite [19]), hematite is a weak ferromagnet due to canting of the antiferromagnetic lattice moments. The Fe(III)-ion spins lie in the basal plane, perpendicular to the $c$-axis of the hematite crystal. Spins are coupled parallel within the same basal plane and coupled antiparallel between adjacent planes. This arrangement leads to a strong magnetocrystalline anisotropy. The magnetic moments in alternating layers are slightly canted, giving rise to a small net magnetization perpendicular to the $c$-axis. At $T_{\mathrm{M}}$, a spin flip transition takes place and the spins align parallel to the $c$-axis, retaining the antiferromagnetic coupling between adjacent basal planes and ferromagnetic coupling within a given plane. At $T<T_{\mathrm{M}}$, hematite is thus antiferromagnetic. These characteristics are affected by particle size and shape. Spherical hematite particles with diameter smaller than 25-30 nm are superparamagnetic [20] and the Morin temperature is lowered significantly, or even suppressed [21]. The Morin transition has been shown to be strongly affected by strain and lattice defects and can be shifted towards bulk values by annealing $[8,21,22]$.

In the present work, we compare the magnetic response of spindle-type hematite particles, synthesized by two previously reported methods [13, 23] with and without silica coating $[15,16]$. The first method, developed by Matijević et al [13], consists in using forced hydrolysis of iron III chloride at elevated temperature in the presence of $\mathrm{HNa}_{2} \mathrm{PO}_{4} \cdot 2 \mathrm{H}_{2} \mathrm{O}$. We label this method M1 in our study. In the work of Ocaña and co-workers [23] hematite particles with larger aspect ratio and smaller volume are obtained using iron perchlorate as a precursor in the presence of $\mathrm{NaH}_{2} \mathrm{PO}_{4} \cdot \mathrm{H}_{2} \mathrm{O}$ and urea to modify the starting $\mathrm{pH}$ of the solution. We refer to this method as M2.

\section{Experimental details}

\subsection{Synthesis}

Iron (III) perchlorate hexahydrate $\mathrm{Fe}\left(\mathrm{ClO}_{4}\right)_{3} \cdot 6 \mathrm{H}_{2} \mathrm{O}$, iron (III) chloride $\mathrm{Fe}(\mathrm{Cl})_{3}$, polyvinylpyrrolidone (PVP, $10 \mathrm{~kg} \mathrm{~mol}^{-1}$ ), and tetramethylammonium hydroxide (TMAH) solution $25 \mathrm{wt} \%$ in water were purchased from Sigma Aldrich and were used as received. Sodium dihydrogen phosphate monohydrate $\mathrm{NaH}_{2} \mathrm{PO}_{4} \cdot \mathrm{H}_{2} \mathrm{O}, \mathrm{HNa}_{2} \mathrm{PO}_{4} \cdot 2 \mathrm{H}_{2} \mathrm{O}$, urea and absolute ethanol from Fluka, and tetraethyl orthosilicate (TEOS) from Merck were also used without further purification. Ultra-pure water (resistivity $=18.2 \mathrm{M} \Omega \mathrm{cm}$ ) purified by a $\operatorname{Milli} Q$ system was used throughout the experiments.

Using method M1, we dissolved $6.47 \mathrm{~g}$ of $\mathrm{Fe}(\mathrm{Cl})_{3}$ and $118 \mathrm{mg}$ of $\mathrm{HNa}_{2} \mathrm{PO}_{4} \cdot 2 \mathrm{H}_{2} \mathrm{O}$ in 2.01 of Milli $Q$ water and aged it for seven days in a preheated oven at $98^{\circ} \mathrm{C}$. The aged solution appeared brownish with a sediment containing the formed particles. Particles were recovered and concentrated by five cleaning steps consisting of centrifugation and redispersion in water. The hematite particles thus obtained were labeled as sample H1. For the second method M2, we dissolved $116 \mathrm{~g}$ of iron (III) perchlorate $\mathrm{Fe}\left(\mathrm{ClO}_{4}\right)_{3} \cdot 6 \mathrm{H}_{2} \mathrm{O}, 1.88 \mathrm{~g}$ of $\mathrm{NaH}_{2} \mathrm{PO}_{4} \cdot \mathrm{H}_{2} \mathrm{O}$ and $15.1 \mathrm{~g}$ of urea in 2.51 of Milli $Q$ water. The ageing was done for $24 \mathrm{~h}$ in a preheated oven at $98{ }^{\circ} \mathrm{C}$. We refer to the resulting particles as $\mathrm{H} 2$.

Parts of the batches $\mathrm{H} 1$ and $\mathrm{H} 2$ were coated with silica according to methods described in the literature $[15,16]$. In the following, we will refer to the silica coated $\mathrm{H} 1$ and $\mathrm{H} 2$ hematite particles as $\mathrm{H} 1 \mathrm{~S}$ and $\mathrm{H} 2 \mathrm{~S}$, respectively. For a typical $20 \mathrm{~nm}$ thick silica coating, about $100 \mathrm{mg}$ of H1 (or H2) were dispersed in $220 \mathrm{ml}$ of Milli $Q$ water, $2 \mathrm{~g}$ of PVP were then added to the suspension and the excess of PVP was removed by centrifuging. Then the PVP stabilized hematite particles were concentrated into $10 \mathrm{ml}$ of water to which $225 \mathrm{ml}$ of absolute ethanol and $85 \mathrm{mg}$ of TMAH $25 \mathrm{wt} \%$ were added. We transfered the suspension into a plastic bottle and stirred mechanically at $400 \mathrm{rpm}$. A mixture of $1 \mathrm{ml}$ of TEOS and $3 \mathrm{ml}$ ethanol was then added in four portions to the stirred suspension every $30 \mathrm{~min}$. Stirring was continued for eight more hours, after which the particles H1S or H2S were washed with water. Particles denoted as H2SS are obtained by coating particles of $\mathrm{H} 2 \mathrm{~S}$ with a second silica coating following the same process.

\subsection{Instrumentation and methods}

X-ray diffraction (XRD) patterns of the particles were measured on a PW1800 (Philips) diffractometer at $40 \mathrm{kV} / 40 \mathrm{~mA}$ using $\mathrm{Cu} \mathrm{K} \alpha$ radiation. The particles were first dispersed in ethanol and subsequently dried on a glass slide. The XRD data were analyzed by Rietveld-refinement (Topas 3, Bruker Inc.) using the fundamental parameter approach [24] and the contribution to specimen line broadening (i.e. crystallite size and strain broadening) was treated by a double-Voigt approach [25]. The value of the crystallite size broadening represents the volume weighted mean column height. The lattice strain is defined as the relative deviation from the average lattice spacing. Transmission electron microscopy (TEM) was performed on a Philips CM100-Biotwin operated at $80 \mathrm{keV}$ and high resolution TEM (HRTEM) on a CM200 at $200 \mathrm{keV}$. For the sample preparation, a droplet of particles dispersed in ethanol was dried onto a carbon coated copper grid. The mean particle dimensions were determined from a statistical 

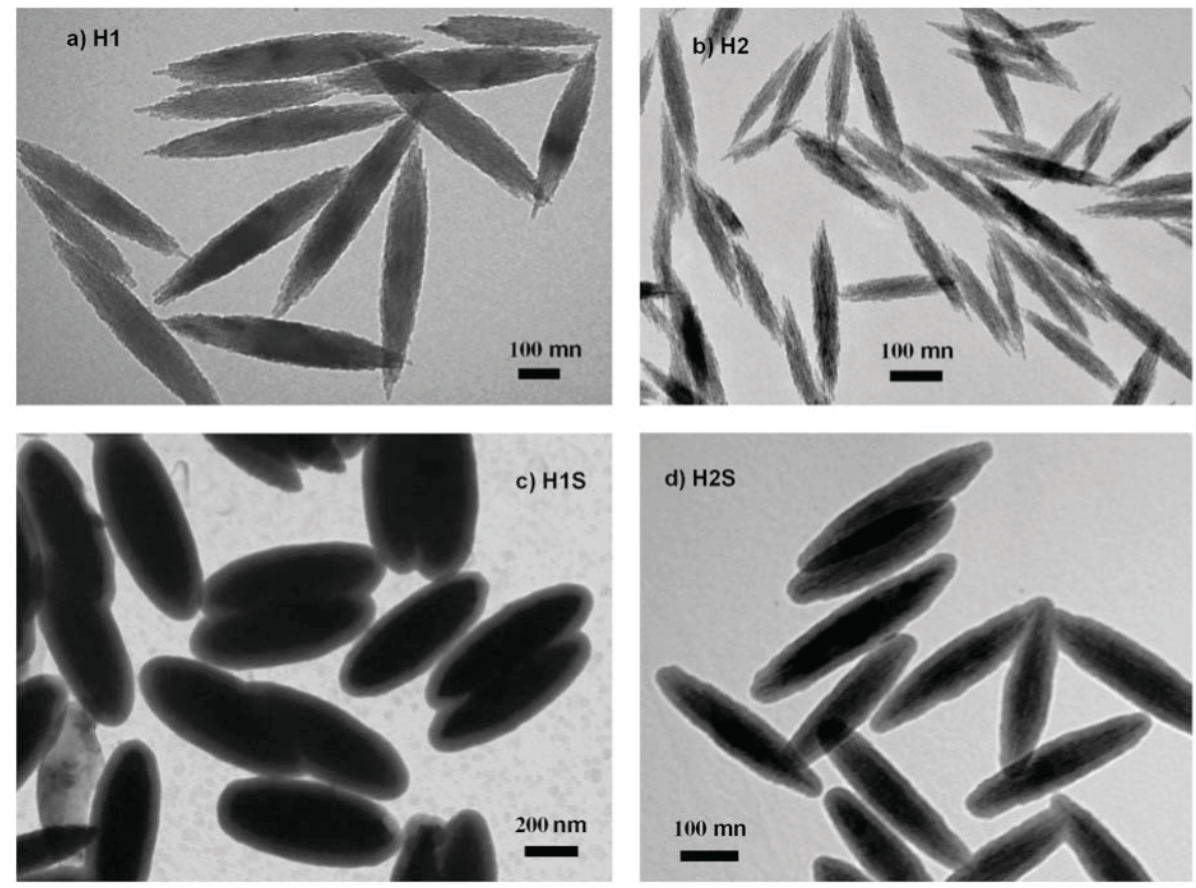

Figure 1. TEM images of hematite particles synthesized by method M1 (H1 and H1S) and method M2 (H2 and H2S).

analysis of TEM images; approximately 200 particle contours were fitted with ellipses corresponding to prolate spheroids of given cross section. Selected area electron diffraction (SAED) patterns were indexed using the JEMS software [26]. Scanning electron microscopy (SEM) was performed on a Philips FEI XL30 Sirion FEG. Before imaging, the samples were sputtered with a $20 \mathrm{~nm}$ thick gold layer. Small-angle X-ray scattering (SAXS) was conducted at the cSAXS beamline at $12 \mathrm{keV}$ at the Swiss Light Source (Paul Scherrer Institute, Villigen, Switzerland). The particles were dispersed in water and measured in $1.0 \mathrm{~mm}$ quartz capillaries. The magnetic field was applied using a water-cooled electromagnet (Buckley Systems Ltd) that was tilted by an angle $10^{\circ}$ relative to the horizontal direction to avoid contributions of slit scattering along the field direction. Magnetization curves on dried powder samples (pressed powder pills with a few mg mass) were measured using a PPMS (Quantum Design QD6000) equipped with a vibrating sample magnetometer (model P525). First-order reversal curves (FORC, [27]) were measured on a vibrating sample magnetometer (PMC model 3900) by applying a field $H_{\mathrm{A}}$ directly after saturating the sample in a $1.0 \mathrm{~T}$ field and recording the magnetization at each intermediate field $H_{\mathrm{B}}$ as the sample was brought back to saturation at $1.0 \mathrm{~T}$. A data-set of the magnetization $M\left(H_{\mathrm{A}}, H_{\mathrm{B}}\right)$ was thus obtained from which the FORC distribution $\rho\left(H_{\mathrm{U}}, H_{\mathrm{C}}\right)$ was calculated from $\rho\left(H_{\mathrm{A}}, H_{\mathrm{B}}\right)=\partial^{2} M\left(H_{\mathrm{A}}, H_{\mathrm{B}}\right) / \partial H_{\mathrm{A}} \partial H_{\mathrm{B}}$ and a change of coordinates to $H_{\mathrm{C}}=\left(H_{\mathrm{B}}-H_{\mathrm{A}}\right) / 2$ and $H_{\mathrm{U}}=\left(H_{\mathrm{B}}+H_{\mathrm{A}}\right) / 2$ using the program FORCinel v. 1.17 [28]. The magnetic torque was measured with a home-built torsion magnetometer [29]. The ac-susceptibility was measured on a PPMS (Quantum Design) equipped with ac-susceptibility and dc-magnetization options at a field strength of $1 \mathrm{mT}$.

\section{Results and discussion}

\subsection{Particle characterization}

An important difference between the two synthesis routes is that the M1 synthesis leads first to primary particles made of $\beta$-FeOOH that aggregate and transform to strongly coalesced hematite spindles whereas the M2 synthesis forms primary hematite particles that aggregate to spindles [14, 23]. Moreover, particles synthesized by method M1 are considerably larger in size than those by method M2 as shown by the mean values for the particle dimensions found by TEM (figure 1 and table 1).

In a first step, we correlate the magnetic properties of the hematite particle with its shape. The Rietveld-refinement of XRD patterns confirms the hematite structure of the particles synthesized with both methods M1 and M2. The $a$ and $c$ lattice parameters (5.036 and $13.787 \AA$ for M1, and 5.035 and $13.787 \AA$ for M2) are slightly larger than those found in the literature for bulk hematite (5.034 and $13.747 \AA$ [30]). This difference can be explained by the small grain size, since the positions of the Bragg peaks are shifted to lower scattering angles for crystallites of the order of a few nm in size [31]. Furthermore, lattice defects can have a greater influence on the lattice parameters in such small crystallites [14]. The crystallite sizes found by XRD are $126 \pm 15 \mathrm{~nm}$ for H1, 23.6土 $1.8 \mathrm{~nm}$ for $\mathrm{H} 2$ and $28.8 \pm 3.4 \mathrm{~nm}$ for $\mathrm{H} 2 \mathrm{~S}$. The significantly larger error for $\mathrm{H} 2 \mathrm{~S}$ is due to the background signal from the amorphous silica coating. The values for the lattice strains are $0.066 \pm 0.01$ for $\mathrm{H} 2$ and $0.105 \pm 0.02$ for $\mathrm{H} 2 \mathrm{~S}$ and have to be considered as important strains. It has been shown that the crystallite size in spindle-type particles of type $\mathrm{H} 2$ increases upon annealing due to coalescence of the sub-units with a size 

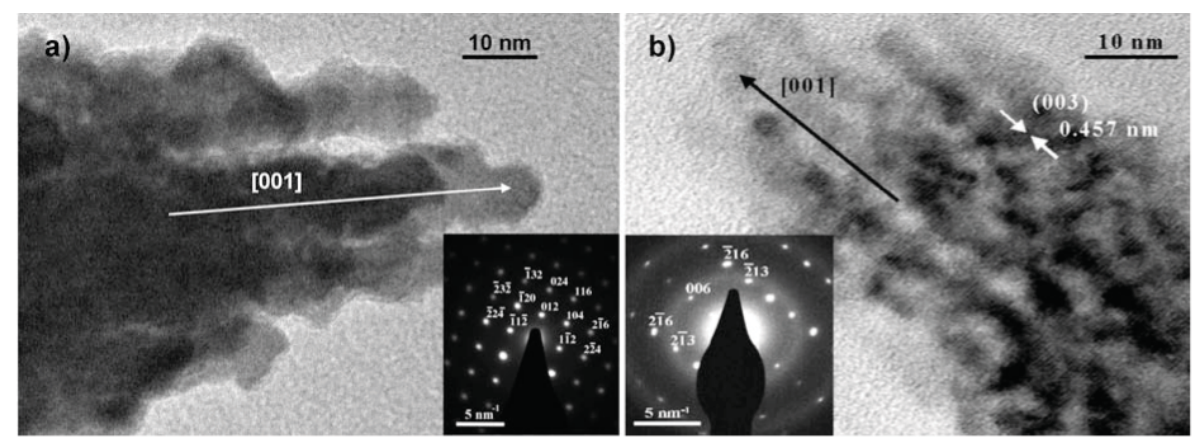

Figure 2. HRTEM image combined with SAED pattern of an $\mathrm{H} 1$ particle (a) and an $\mathrm{H} 2$ particle (b). In (a) the arrow corresponds to the projected direction [001], whereas in (b) the direction [001] is lying in the image plane. The smeared out diffraction spots in (b) (e.g. -216) point to misorientation between adjacent domains.

Table 1. Parameters obtained from the TEM images (mean values for the semi-axes $a$ and $b$ with polydispersity, shell thickness $s$, hematite mass fraction $\left.\frac{m_{\text {hem }}}{m_{\text {part }}}\right)$ and from magnetization curves at $300 \mathrm{~K}$ (normalization factor $\alpha$, remanent magnetization $\sigma_{\mathrm{r}}$, coercivity $H_{\mathrm{C}}$, saturation magnetization $\sigma_{\mathrm{s}}$ and high field susceptibility $\chi^{\mathrm{HF}}$ ).

\begin{tabular}{llcllllllll}
\hline & $a(\mathrm{~nm})$ & $b(\mathrm{~nm})$ & Poly $(\%)$ & $s(\mathrm{~nm})$ & $\frac{m_{\mathrm{hem}}}{m_{\text {part }}}$ & $\alpha$ & $\begin{array}{l}\sigma_{\mathrm{r}} \\
\left(\mathrm{A} \mathrm{m}^{2} \mathrm{~kg}^{-1}\right)\end{array}$ & $\begin{array}{l}H_{\mathrm{C}} \\
\left(\mathrm{kA} \mathrm{m}^{-1}\right)\end{array}$ & $\begin{array}{l}\sigma_{\mathrm{s}} \\
\left(\mathrm{A} \mathrm{m}^{2} \mathrm{~kg}^{-1}\right)\end{array}$ & $\begin{array}{l}\chi^{\mathrm{HF}} \\
\left(10^{-7} \mathrm{~m}^{3} \mathrm{~kg}^{-1}\right)\end{array}$ \\
\hline H1 & 276 & 69 & 25 & 0.0 & 1 & 1 & 0.22 & 36 & 0.32 & 2.5 \\
H1S & 319 & 112 & 20 & 43 & 0.56 & 0.62 & 0.23 & 72 & 0.32 & 2.7 \\
H2 & 154 & 28.9 & 15 & 0.0 & 1 & 1 & 0.030 & 24 & 0.21 & 3.1 \\
H2S & 180 & 42.1 & 10 & 13.2 & 0.67 & 0.49 & 0.008 & 0.8 & 0.21 & 2.8 \\
H2SS & 202 & 61.6 & 9.0 & 33 & 0.37 & 0.22 & 0.004 & 0.8 & 0.21 & 2.8 \\
\hline
\end{tabular}

of the order of $5 \mathrm{~nm}[8]$ and it has been proposed that sub-units in the spindle-type particles are separated by layers of water, $\mathrm{OH}$ radicals and phosphate groups [14].

Figure 2(a) shows the HRTEM image and the SAED pattern taken from a single particle of batch H1. The HRTEM image reveals a fibrous structure at the tip of the particle with a typical distance between the individual fibers of the order of $10 \mathrm{~nm}$. The corresponding HRTEM image for an H2 particle in figure 2(b) shows a similar structure, however the typical distance between the individual fibers is of the order of $5 \mathrm{~nm}$ only. Moreover, the $\mathrm{H} 2$ particle appears considerably less compact than the $\mathrm{H} 1$ particle, the HRTEM image shows darker and brighter sub-units as also observed by Ocaña et al [23].

Both SAED patterns in figure 2 indicate that the crystalline orientation of the sub-units is the same throughout a spindletype particle and the particles behave crystallographically as single crystals in agreement with findings from other groups $[8,14,23]$. The lattice fringes in the HRTEM images cross the entire spindles, i.e. also the brighter areas, which confirms the single-crystalline nature of the particles. However, direct comparison between the SAED patterns of the $\mathrm{H} 1$ and $\mathrm{H} 2$ particles reveals that the spots of the $\mathrm{H} 2$ particle are considerably more smeared out, particularly at high scattering angles. This suggests, in combination with the comparison of the HRTEM images, that the synthesis M2 leads to more porous particles with substantially more lattice defects compared to the M1 synthesis.

For both types of particles, the sub-units observed by TEM are considerably smaller than the crystallite sizes obtained by XRD. Similar observations have been made on a goethite system and associated with oriented attachment of the adjacent units, such that the crystalline order continues to some extent across grain boundaries [32]. Another reason for the difference might be that XRD reveals the volume weighted average and thus values shifted towards the upper tail of a broad grain distribution.

\subsection{Particle orientation in a magnetic field}

The indexing of the SAED patterns in figure 2 reveals that the $c$-axis [001] runs parallel to the major particle axis (hexagonal unit cell representation). As mentioned above, the net magnetic moment of hematite lies in the basal (easy) plane above the Morin temperature; therefore spindles will align with the major axis perpendicular to an external magnetic field. To confirm this assumption we carried out SAXS on diluted $(0.2 \mathrm{wt} \%)$ aqueous hematite particle dispersions in an external magnetic field. Figure 3 shows scattering patterns of dispersed particles in a magnetic field along the indicated direction. At low magnetic field $(6 \mathrm{mT})$ the sample $\mathrm{H} 2 \mathrm{~S}$ shows isotropic orientation whereas sample H1S is already aligned. At high field (1 $\mathrm{T})$ both samples show a scattering pattern that is elongated along the field direction. This corresponds to a particle orientation where the major axis is perpendicular to the field in the detector plane. However, with this configuration particles are also allowed to orient parallel to the beam, which leads to a symmetrical background.

In a second step, we prepared films of particles by applying a magnetic field during the drying process. The particles were dispersed in ethanol and dried at room 


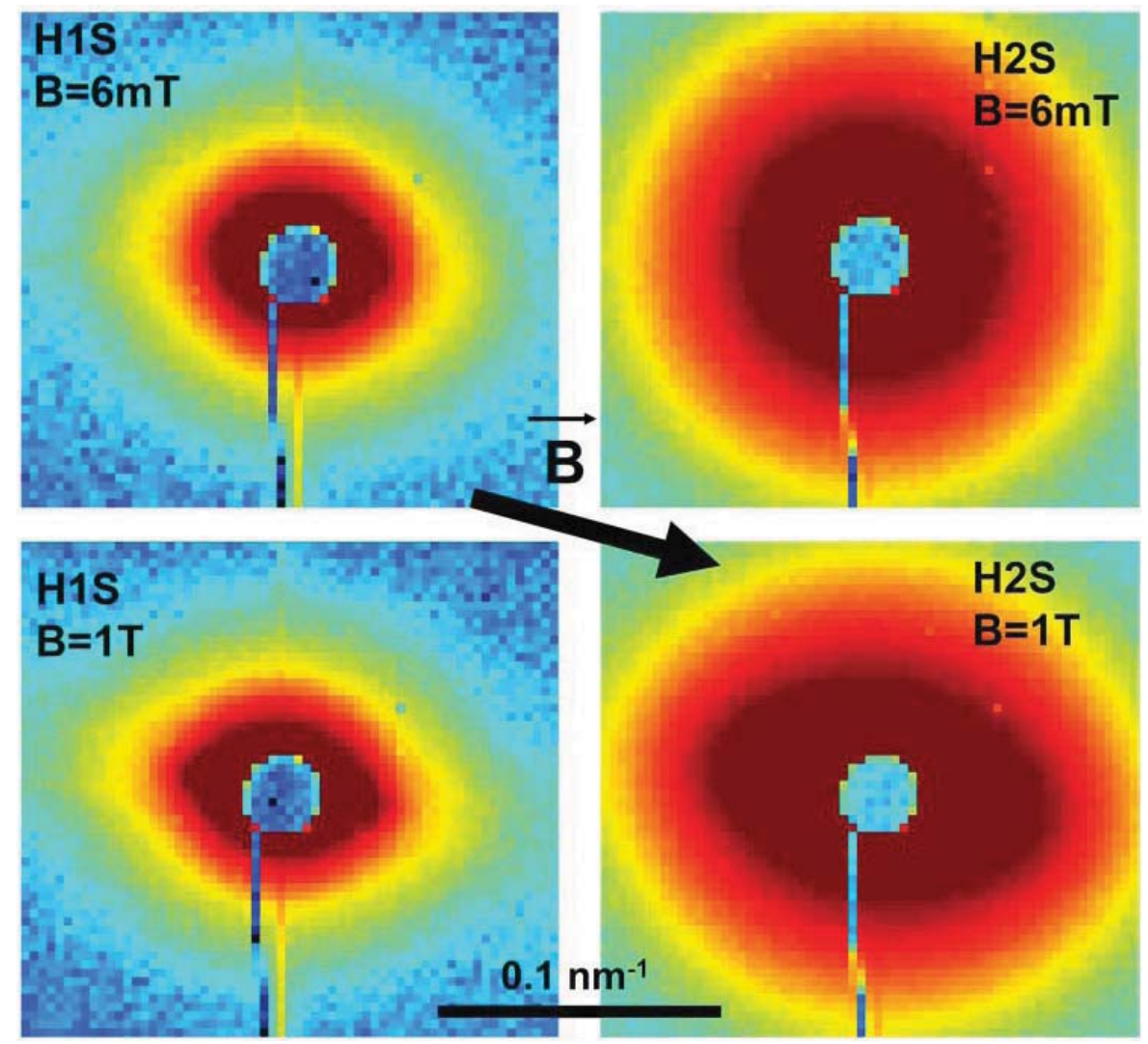

Figure 3. SAXS scattering patterns obtained from hematite particles dispersed in water in a magnetic field along the indicated direction.

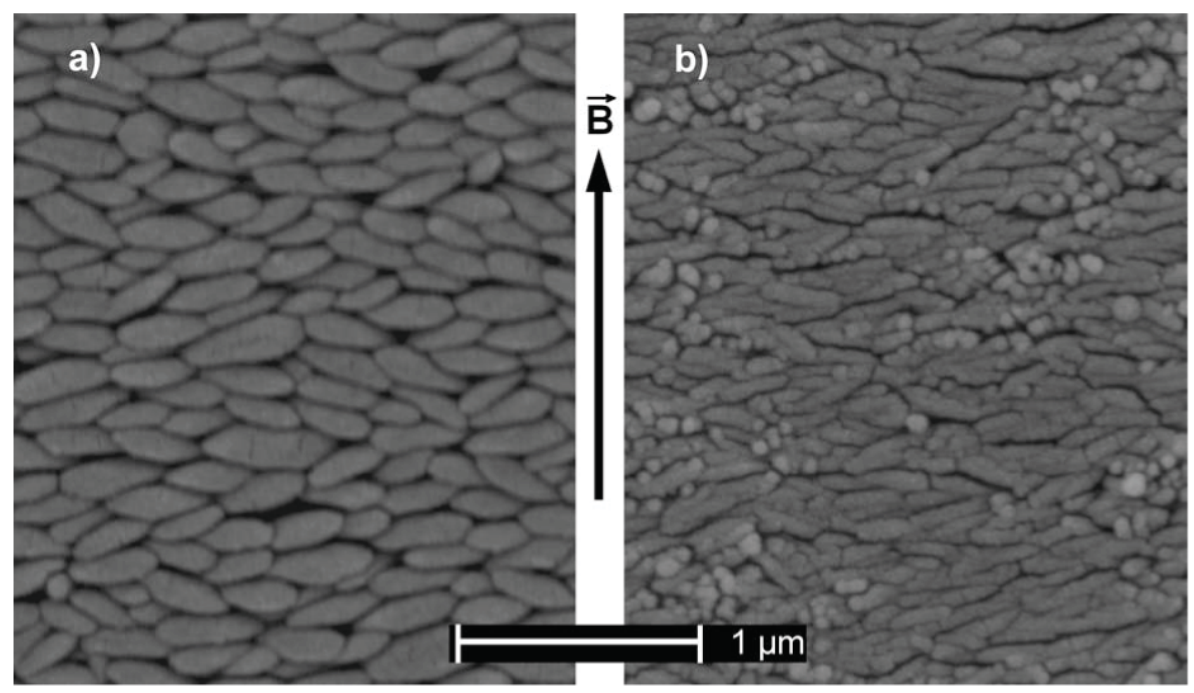

Figure 4. SEM images of films of hematite particles H1S (a) and H2 (b) where a magnetic field of approximately $0.7 \mathrm{~T}$ in the indicated direction has been applied during the drying process. In both cases the particles preferentially orient perpendicular to the direction of the magnetic field.

temperature on a carbon support mounted between two permanent magnets resulting in a homogeneous magnetic field of approximately $0.7 \mathrm{~T}$. Figure 4 shows that in this way most particles align with their major axis in a plane perpendicular to the magnetic field applied during the drying process. The additional preferential alignment into a single direction when compared to the dispersions (figure 3 ) is probably due to a combination of the magnetic field, the formation of nematic ordering, and the thin film geometry of the sample. The alignment of particles synthesized by method M1 was generally better, indicating a stronger magnetic response. This observation is consistent with the SAXS study where particles of batch H1S appear to orient more readily in small magnetic fields. 

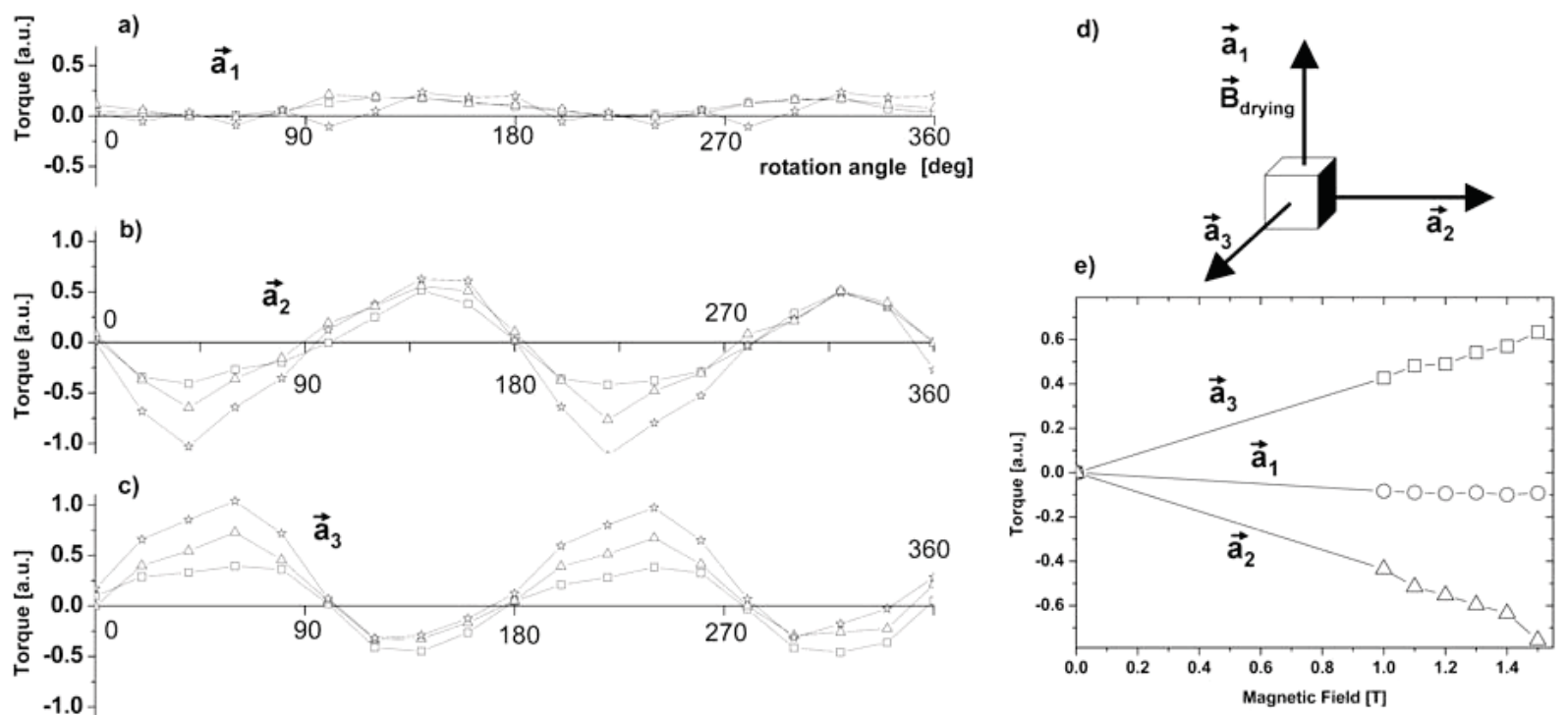

Figure 5. (a)-(c) Torque curves of sample H1S for different fields (squares: 1.0 T, triangles: 1.2 T, stars: $1.5 \mathrm{~T}$ ) when turned around the axes $\vec{a}_{1}, \vec{a}_{2}$ and $\vec{a}_{3}$ as defined in sketch (d). The units are arbitrary but the same for (a)-(c). The measured torque is proportional to the applied magnetic field as can be seen in (e) where the torque amplitudes are plotted for the different fields.

The anisotropic particle orientation can be shown directly by measuring the torque due to the magnetic moment in a field, since no torque would arise from an isotropic magnetization. Figures 5(a)-(c) show the torque signals of a sample of particles H1S (prepared similarly to those for the SEM study) when the sample is rotated around the three axes $\vec{a}_{1}, \vec{a}_{2}$ and $\vec{a}_{3}$ defined in figure 5(d) with applied magnetic field perpendicular to the rotation axis. When rotated around $\vec{a}_{1}$, no significant torque arises due to the uniaxial nature of anisotropy for hematite. In contrast, when rotated around $\vec{a}_{2}$ or $\vec{a}_{3}$, the torque shows the periodic signal representing the magnetocrystalline anisotropy arising from the preferential particle orientation. Considering the accuracy of particle alignment, there is a clear linear dependence of the torque with field as expected for hematite, as shown in figure 5(e). Thus, the bulk magnetic moment has no significant paramagnetic contributions, which would show a dependence with the square of the field. These experiments identify the basal plane as the easy plane of magnetization on a single particle level for the investigated hematite particles.

\subsection{Magnetic properties of dried, randomly oriented particles}

In a next step we examined the magnetic properties of randomly oriented particles in powder form. Magnetization curves were measured to obtain the saturation magnetization $\sigma_{\mathrm{s}}$ and the high field susceptibility $\chi^{\mathrm{HF}}$. This was done by fitting the magnetization data in the range 1.5-2.2 $\mathrm{T}$ with $\alpha\left(\sigma_{\mathrm{s}}+H \chi^{\mathrm{HF}}\right)$, where we set $\alpha=1$ for the uncoated particles (H1 and H2). For the silica coated samples, the parameter $\alpha$ accounts for the effective mass content of hematite and is chosen such that $\sigma_{\mathrm{s}}$ matches the value of the corresponding uncoated particles. We therefore assume that $\sigma_{\mathrm{s}}$ is not affected by a silica coating. The fitted values for $\sigma_{\mathrm{s}}, \chi^{\mathrm{HF}}$ and $\alpha$ are listed in table 1 . The values for $\chi^{\mathrm{HF}}$ are in good agreement with the value of $2.5 \times 10^{-7} \mathrm{~m}^{3} \mathrm{~kg}^{-1}$ for bulk hematite found in the literature [33]. The values of $\sigma_{\mathrm{s}}$ are lower than typically reported for bulk hematite $\left(0.4 \mathrm{~A} \mathrm{~m}^{2} \mathrm{~kg}^{-1}\right.$ [33]), but still within the range of values reported in the literature. To justify the values for $\alpha$, we calculate the hematite mass fraction $m_{\text {hem }} / m_{\text {part }}$ based on a silica density of $2.0 \mathrm{~g} \mathrm{~cm}^{-3}$, a hematite density of $5.26 \mathrm{~g} \mathrm{~cm}^{-3}$ and the particle dimensions listed in table 1. For both samples the values found for $\alpha$ are in good agreement with the calculated values for $m_{\text {hem }} / m_{\text {part }}$ as shown in table 1 . In the case of the H2S and H2SS particles, the porous hematite core [34] might lead to an overestimation of $m_{\text {hem }} / m_{\text {part }}$, which further improves the agreement.

We use the above values for $\alpha$ to rescale the magnetization data and obtain the hysteresis curves for particles synthesized by methods $\mathrm{M} 1$ and $\mathrm{M} 2$ as shown in figures 6 and 7 , respectively. For both methods, the rescaling leads to a reasonable superposition of the hysteresis curves at high fields, which means that $\chi^{\mathrm{HF}}$ is not affected by the silica coating and is a bulk property originating from antiferromagnetism [33]. Particles synthesized by method M1 show hysteresis curves as expected for single domain (SD) particles. The silica coated particles (H1S in figure 6(a)) have an increased coercivity $H_{\mathrm{C}}$ compared to the uncoated particles but the remanent magnetization $\sigma_{\mathrm{r}}$ remains unchanged. At lower temperature $H_{\mathrm{C}}$ is further increased as shown in figure 6(b). However, the effect of the silica coating is far more significant for particles synthesized by M2. Both, $H_{\mathrm{C}}$ and $\sigma_{\mathrm{r}}$ are reduced drastically by the silica coating (figure $7(\mathrm{a})$ ) and the hysteresis curves collapse to a curve similar to what is observed for superparamagnetic (SP) particles. Moreover, at low temperature the hysteresis of the silica coated particles is recovered as shown in figure 7(b). Such a fundamentally different behavior of the particles synthesized by M1 and M2 is unexpected. In particular, the observed influence of the silica coating on particles synthesized by M2 is surprising. 


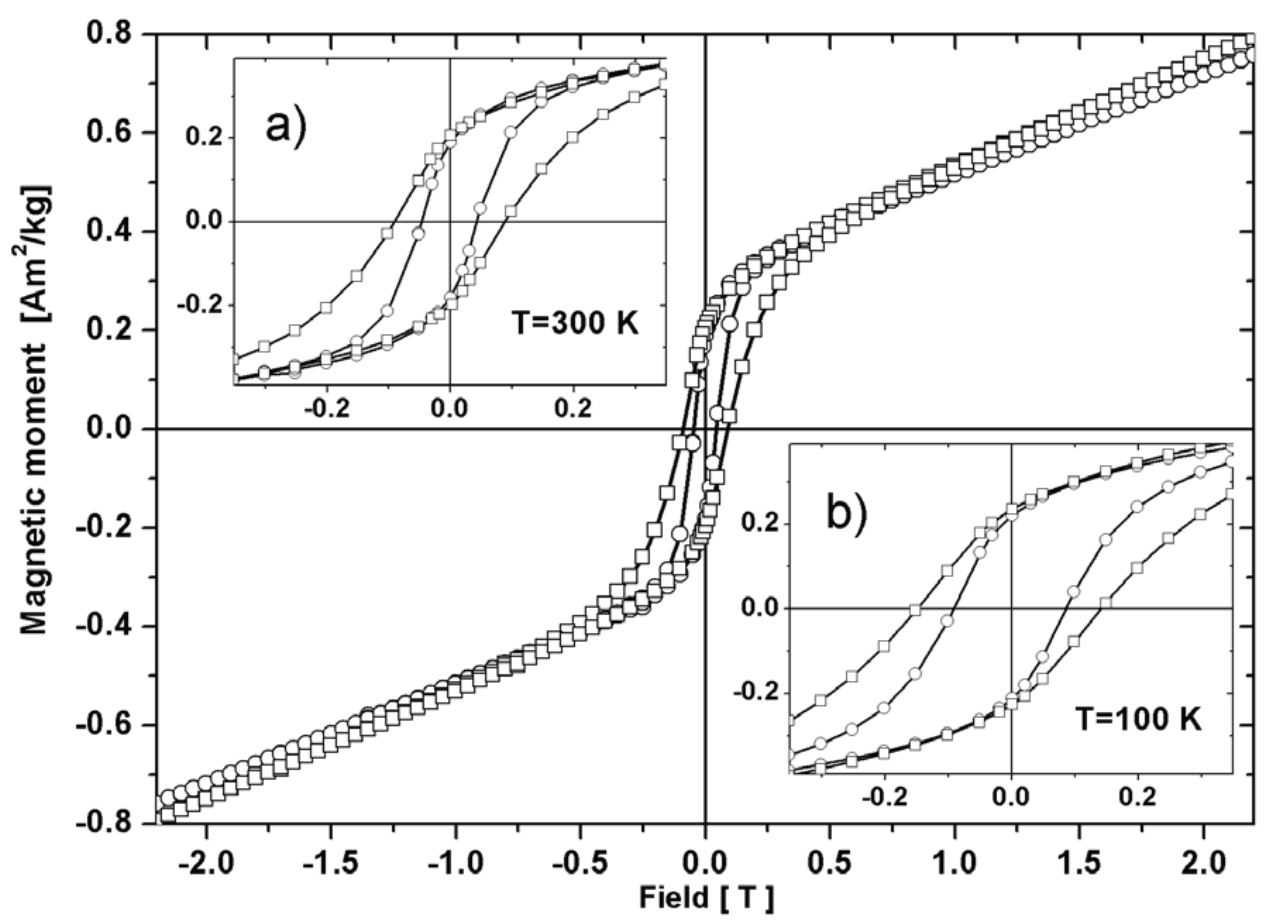

Figure 6. Magnetization curves of particles synthesized by M1 without silica coating H1 (circles) and with silica coating H1S (squares). The insets show the low field data at temperatures of $300 \mathrm{~K}$ (a) and $100 \mathrm{~K}$ (b). The data are normalized by the factor $\alpha$ (table 1).

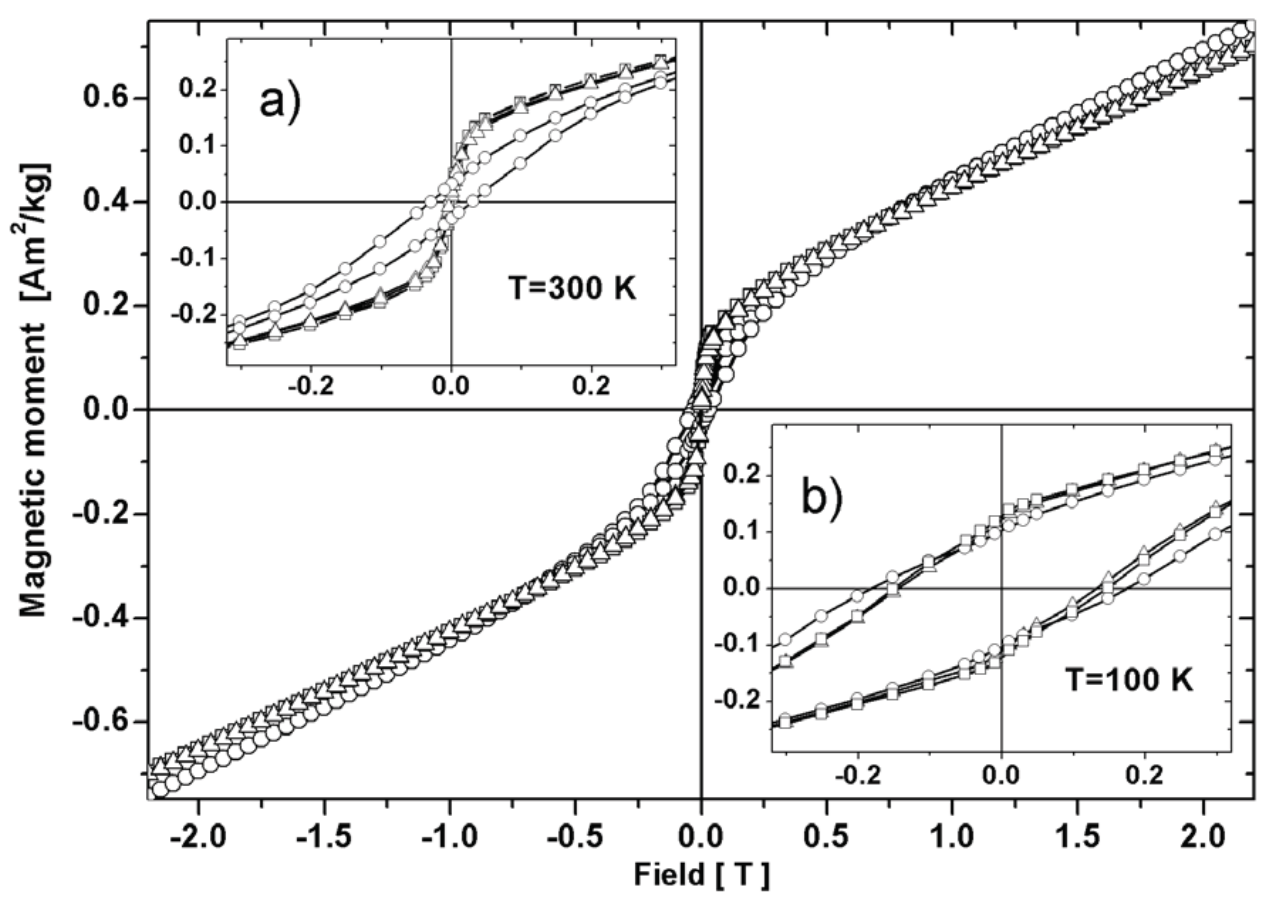

Figure 7. Magnetization curves of particles synthesized by M2 without silica coating $\mathrm{H} 2$ (circles), with silica coating H2S (squares) and with thick silica coating H2SS (triangles). The insets show the low field data at temperatures of $300 \mathrm{~K}$ (a) and $100 \mathrm{~K}$ (b). The data are normalized by the factor $\alpha$ (table 1$)$.

\subsection{FORC measurements}

In order to investigate the differences between the particles synthesized by M1 and M2 with respect to thermal relaxations and magnetostatic interactions we use FORC diagrams [35]. As shown in figures 8(a) and (b) the FORC distributions for $\mathrm{H} 1$ and H1S both show a broad coercivity distribution between 10 and $200 \mathrm{mT}$, which peaks around $50 \mathrm{mT}$. Such distributions 

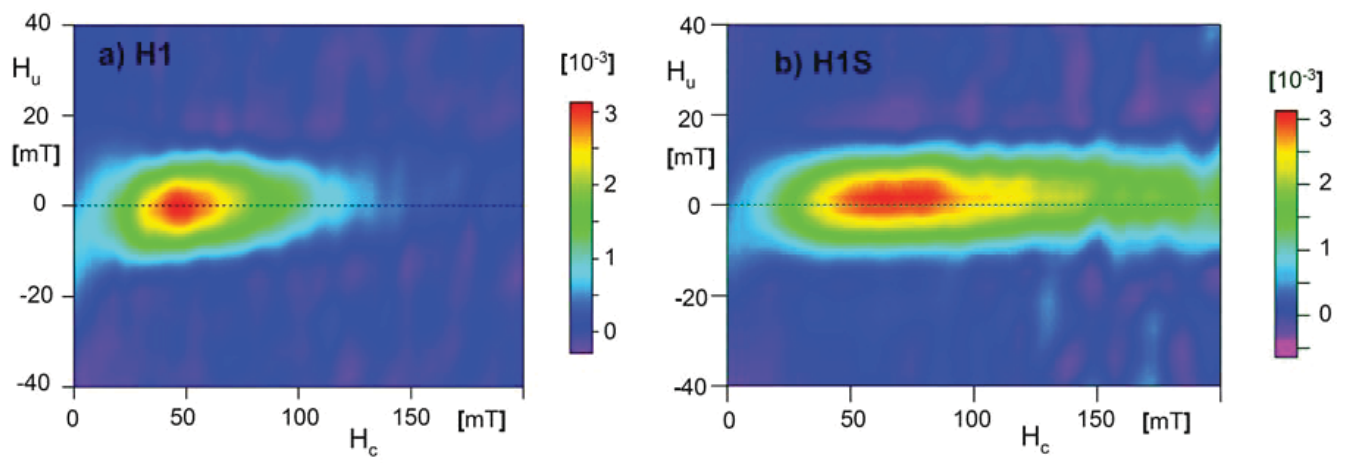

Figure 8. FORC diagrams (interaction field $H_{\mathrm{U}}$ as a function of the coercivity $H_{\mathrm{C}}$ ) for particles prepared by method M1 without (a) and with (b) silica coating.
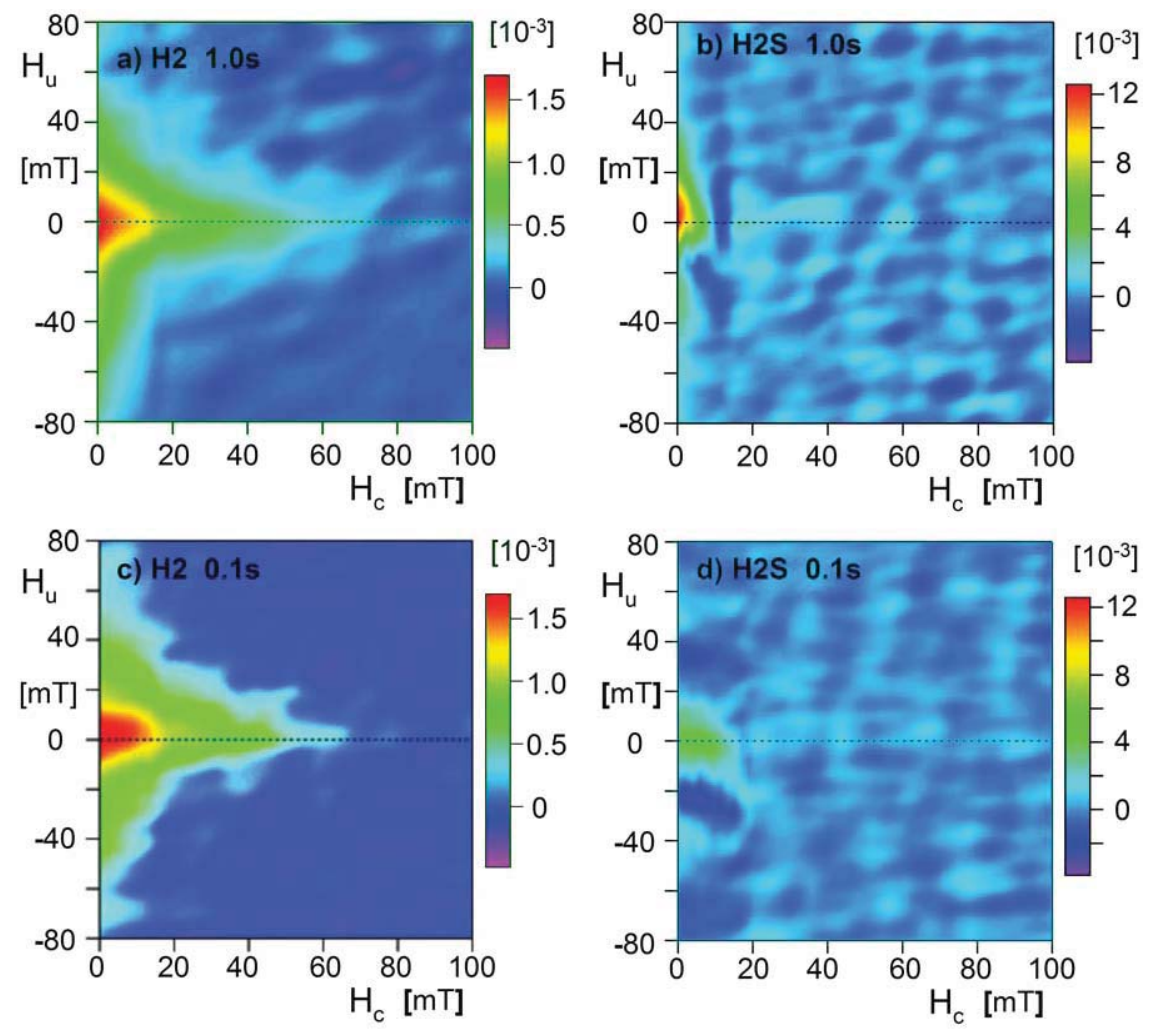

Figure 9. FORC diagrams (interaction field $H_{\mathrm{U}}$ as a function of the coercivity $H_{\mathrm{C}}$ ) for particles prepared by method M2 measured with $1.0 \mathrm{~s}$ averaging time ((a), (b)) and with $0.1 \mathrm{~s}$ averaging time ((c), (d)).

are typical for hematite SD particles [36]. The shifted peak towards higher values of $H_{\mathrm{C}}$ and the broader distribution found in the FORC distribution for H1S can result from increased lattice strain [37] or be related to a different distribution of the effective SD-grain size [38].

The FORC distributions for $\mathrm{H} 2$ and $\mathrm{H} 2 \mathrm{~S}$ in figures 9(a) and (b) are centered at very low coercivity $H_{\mathrm{C}}$, as would be expected for an assemblage of grains that are on the boundary between SD and SP. The onset of SP at room temperature is associated with a grain size of 25-30 nm [20], which is in agreement with the crystallite size found by XRD. In particular H2S particles show all the features related to SP [39]: a peak at the origin with vertical tails and slightly shifted towards positive values of $H_{\mathrm{U}} . \mathrm{H} 2$ has a larger distribution of $H_{\mathrm{C}}$, indicating a broader range of effective grain sizes compared to $\mathrm{H} 2 \mathrm{~S}$. The FORC distribution for $\mathrm{H} 2 \mathrm{~S}$ is confined both in terms of coercivity and interaction field, which reflects a reduction of interactions between the grains compared to $\mathrm{H} 2$. This confirms the assumption that the magnetic properties of the particles synthesized by M2 are governed by grains much smaller than the spindle size since compact hematite particles with size of the order of the spindle show SD behavior [20].

To further investigate the relaxation behavior of the particles synthesized by M2 we measure the same samples 

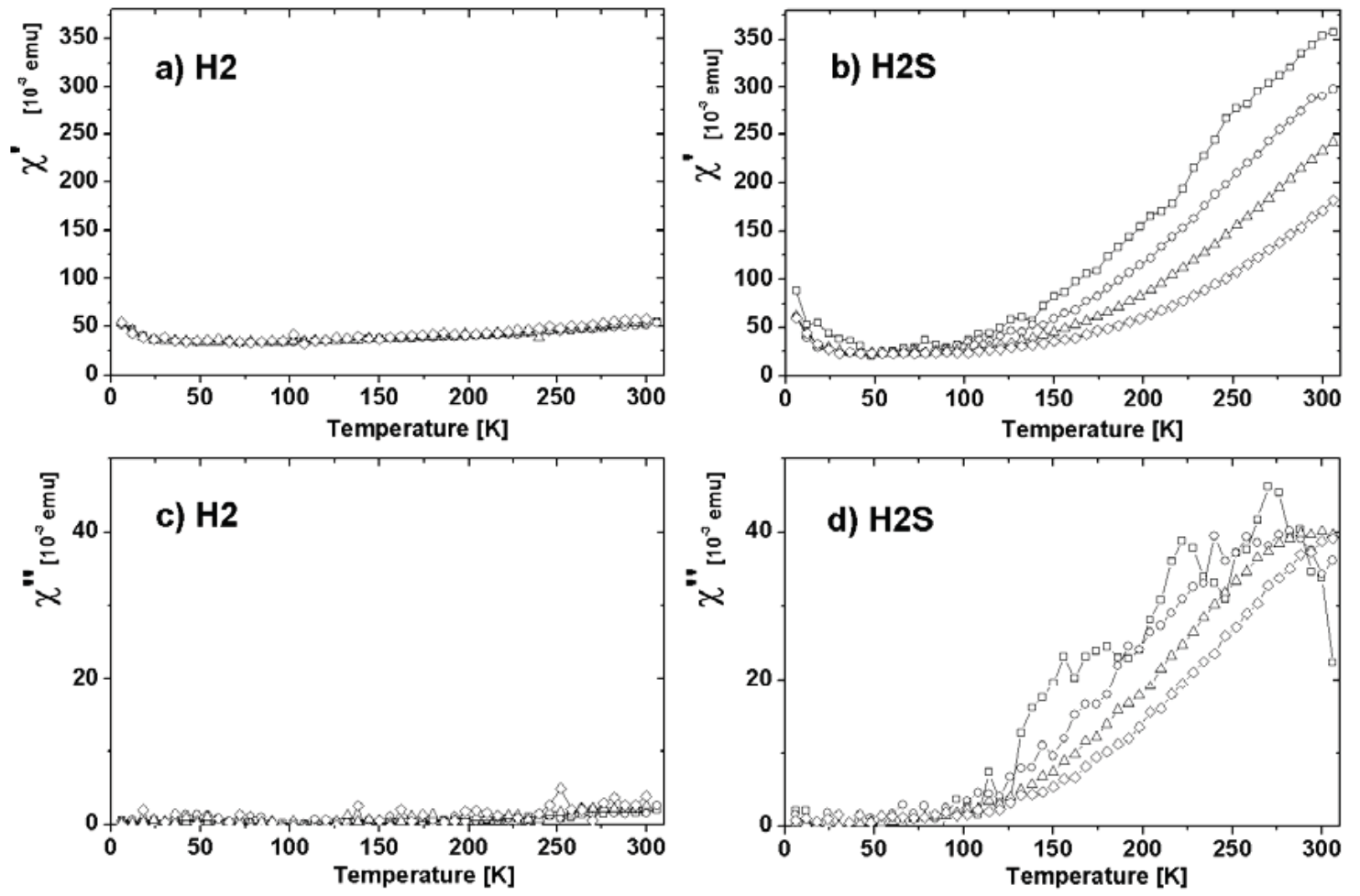

Figure 10. In-phase $\chi^{\prime}$ and out-of-phase $\chi^{\prime \prime}$ ac-susceptibility measured with samples $\mathrm{H} 2$ and $\mathrm{H} 2 \mathrm{~S}$ at different frequencies (squares: $10 \mathrm{~Hz}$, spheres: $100 \mathrm{~Hz}$, triangles: $1 \mathrm{kHz}$, diamonds: $10 \mathrm{kHz}$ ).

using a shorter averaging time $(0.1 \mathrm{~s}$ instead of $1 \mathrm{~s})$, i.e. a faster measurement, as shown in figures 9(c) and (d). The direct comparison of figures 9(c) and (d) with (a) and (b), respectively, reveals a shift of the peak in $H_{\mathrm{C}}$ towards higher values whereas the peak at the origin, representing the SP contribution, has disappeared. This indicates that the faster measurement is able to catch more particles before they relax, leading to a more SD-type of FORC distribution. This demonstrates that relaxation in the $\mathrm{H} 2 \mathrm{~S}$ particles is occurring on the scale of the measurement time of $0.1 \mathrm{~s}$.

\section{5. ac-susceptibility}

To confirm the superparamagnetic character of the particles synthesized by M2 we perform ac-susceptibility measurements. Figure 10 shows the in-phase $\chi^{\prime}$ and out-of-phase $\chi^{\prime \prime}$ ac-susceptibility of the samples $\mathrm{H} 2$ and H2S. Sample H2 shows very little frequency dependence in both $\chi^{\prime}$ and $\chi^{\prime \prime}$ except close to $300 \mathrm{~K}$. The rise in $\chi^{\prime}$ and $\chi^{\prime \prime}$ approaching $300 \mathrm{~K}$ indicates that the average blocking temperature $T_{\mathrm{B}}$ is above room temperature. In contrast, the $\mathrm{H} 2 \mathrm{~S}$ particles appear to peak close to $300 \mathrm{~K}$ with frequency dependent position and height for both $\chi^{\prime}$ and $\chi^{\prime \prime}$. The peak position of $\chi^{\prime \prime}$ is shifted with increasing frequency $f$ to higher temperatures reflecting the higher activation energy $k_{\mathrm{B}} T$ required for relaxation on a timescale of the order of $1 / f$.

This is a clear sign of superparamagnetic relaxation and, along with the FORC distributions, suggests that the M2 synthesis method leads to particles with small magnetic domains within the spindles that are only partially exchange coupled. The faster relaxation of the $\mathrm{H} 2 \mathrm{~S}$ particles compared to the $\mathrm{H} 2$ particles indicates that the exchange coupling between the grains is more disturbed. This is further supported by the reduced interactions in $\mathrm{H} 2 \mathrm{~S}$ compared to $\mathrm{H} 2$ found by FORC. The increased value for the lattice strain (found by XRD) of $\mathrm{H} 2 \mathrm{~S}$ compared to $\mathrm{H} 2$ further suggests that the silica coating leads to mechanical stress that weakens the interactions.

\section{Conclusion}

We have shown by two independent methods (SEM and SAXS) that spindle-type hematite particles synthesized with two different synthesis methods (M1 and M2) align with the major axis perpendicular to an applied magnetic field. This behavior demonstrates that the magnetization is not governed by particle shape as suggested previously [40], but by the magnetocrystalline anisotropy. With torque experiments we identified the basal plane as the easy plane of magnetization, as expected for bulk hematite.

Remarkably, the two synthesis methods result in particles with fundamentally different magnetic properties. Our measurements of hysteresis curves, FORC and acsusceptibility show that particles synthesized by method M1 behave as single domain particles, whereas M2 yields particles behaving magnetically as clusters of interacting superparamagnetic sub-units. This must be related to the aggregation process of sub-units of the order of $5 \mathrm{~nm}$ that differs for the two methods, although both yield spindle particles with essentially single crystal structure [14, 23]. 
With method M1, the sub-units are strongly linked and, therefore, magnetic single domain particles are obtained. In contrast, M2 yields sub-units that appear to be partly isolated (i.e. not exchange coupled) due to voids, excess water [14] and by slight misalignment, although the subunits have the same general crystal orientation. The M2 particles behave as clusters of superparamagnetic nanoparticles with mean blocking temperature $T_{\mathrm{B}}>350 \mathrm{~K}$, as shown by the ac-susceptibility measurements. A partial suppression of superparamagnetic relaxation due to exchange coupling in clusters of iso-aligned hematite nanoparticles was also reported by Frandsen et al [41]. The same authors suggest that exchange coupling favors self-assembly of hematite nanoparticles along their crystalline $c$-axis, which might explain why the subunits iso-align in the synthesis M2. An interaction dependent relaxation behavior was also observed for other systems of antiferromagnetic particles. Madsen et al [32] found for goethite particles that defects lead to magnetic mismatch at the grain boundaries and weaken the magnetic coupling between the grains such that the sublattice magnetization directions can fluctuate.

Interestingly, a silica coating has a clear effect on the magnetic properties of the spindle-type particles obtained by methods M1 and M2. Whereas particles synthesized by M1 show an increased coercivity $H_{\mathrm{C}}, \mathrm{M} 2$ yields particles with a drastically decreased $H_{\mathrm{C}}$ when coated with silica. To our knowledge, such an effect has never been reported, but is in agreement with observations made on the orientational behavior of dispersed particles under dilute conditions in a magnetic field [34]. The increased lattice strain found after silica coating suggests that mechanical stress caused by the coating weakens the interactions between the sub-units. In the case of the considerably smaller and more porous particles obtained by M2, this leads to magnetic domains that are small enough to show superparamagnetic relaxation at room temperature, i.e. smaller than 25-30 nm [20]. In contrast, M1 yields particles which are less affected by the silica coating due to their larger size and more compact structure, and show only an increased $H_{\mathrm{C}}$ that can result from higher lattice strain [37] or a changed grain size distribution [42, 43].

These findings underline the importance of the particle morphology and interactions between their grains for the magnetic properties of nano-sized hematite particles and explain the wide range of magnetic properties reported in the literature.

\section{Acknowledgments}

This work is supported by the Swiss National Science Foundation, COST D43 and the Adolphe Merkle Foundation. The authors also thank Christoph Neururer for SEM facility assistance, Michalis Charilaou for the ac-susceptibility measurements, and Andreas Menzel for help with SAXS measurements. The SAXS experiments were performed on the cSAXS beamline at the Swiss Light Source, Paul Scherrer Institut, Villigen, Switzerland.

\section{References}

[1] Sacanna S, Rossi L and Philipse A P 2007 J. Phys.: Condens. Matter 19376108

[2] Yethiraj A and van Blaaderen A 2003 Nature 421 513-7

[3] Shukoor M I, Natalio F, Tahir M N, Ksenofontov V, Therese H A, Theato P, Schröder H C, Müller W E G and Tremel W 2007 Chem. Commun. 44 4677-9

[4] Liu L-H, Dietsch H, Schurtenberger P and Yan M 2009 Bioconjug. Chem. 20 1349-55

[5] Hilger I, Hergt R and Kaiser W A 2005 J. Magn. Magn. Mater. 293 314-9

[6] Mørup S, Madsen D E, Frandsen C, Bahl C R and Hansen M F 2007 J. Phys.: Condens. Matter 19213202

[7] Mietra S, Das S, Basu S, Sahu P and Mandal K 2009 J. Magn. Magn. Mater. 321 2925-31

[8] Mansilla M V, Zysler R, Fiorani D and Suber L 2002 Physica B 320 206-9

[9] Shi J-B, Lee C-W, Guo J-W, Cheng M-J, Wu C, Chen C-J, Chen Y-C, Lin Y-T and Chang C-C 2007 Mater. Lett. $615268-70$

[10] Muench G J, Arajs S and Matijević E 1981 J. Appl. Phys. 52 2493-5

[11] Suber L, Fiorani D, Imperatori P, Foglia S, Montone A and Zysler R 1999 Nanostruct. Mater. 11 797-803

[12] Raming T P, Winnubst A J A, Kats C M and Philipse A P 2002 J. Colloid Interface Sci. 249 346-50

[13] Ozaki M, Kratohvil S and Matijević E 1984 J. Colloid Interface Sci. 102 146-51

[14] Ocaña M, Morales M P and Serna C J 1995 J. Colloid Interface Sci. 171 85-91

[15] Graf C, Vossen D L, Imhof A and Blaaderen A 2003 Langmuir 19 6693-700

[16] Sacanna S, Rossi L, Kuipers B W M and Philipse A P 2006 Langmuir 22 1822-7

[17] Sánchez-Ferrer A, Reufer M, Mezzenga R, Schurtenberger P and Dietsch H 2010 Nanotechnology 21185603

[18] Dagallier C, Dietsch H, Schurtenberger P and Scheffold F 2010 Soft Matter 6 2174-7

[19] Morrish A H 1994 Canted Antiferromagnetism: Hematite (Singapore: World Scientific)

[20] Dunlop D J and Özdemir Ö 1997 Rock Magnetism: Fundamentals and Frontiers (Cambridge: Cambridge University Press)

[21] Muench G J, Arajs S and Matijević E 1985 Phys. Status Solidi a 92 187-92

[22] Suber L, Santiago A G, Fiorani D, Imperatori P, Testa A M, Angiolini M, Montone A and Dormann J L 1998 Appl. Organomet. Chem. 12 347-51

[23] Ocaña M, Morales M P and Serna C J 1999 J. Colloid Interface Sci. 212 317-23

[24] Cheary R W and Coelho A A 1992 J. Appl. Crystallogr. 25 109-21

[25] Balzar D 1999 Microstructure Analysis from Diffraction ed R L Snyder, H J Bunge and J Fiala (New York: Oxford University Press) pp 94-126

[26] Stadelmann P 1987 Ultramicroscopy 21 131-45

[27] Roberts A P, Pike C R and Verosub K L 2000 J. Geophys. Res. 105 28461-75

[28] Harrison R J and Feinberg J M 2008 Geochem. Geophys. Geosyst. 9 Q05016

[29] Bergmüller F, Barlocher C, Geyer B, Grieder M, Heller F and Zweifel P 1994 Meas. Sci. Technol. 5 1466-70

[30] Finger L W and Hazen R M 1980 J. Appl. Phys. 51 5362-7

[31] Palosz B, Stelmakh S, Grzanka E, Gierlotka S and Palosz W 2007 Z. Kristallogr. 222 580-92

[32] Madsen D E, Cervera-Gontard L, Kasama T, Dunin-Borkowski R E, Koch C B, Hansen M F, 
Frandsen C and Mørup S 2009 J. Phys.: Condens. Matter 21016007

[33] O’Reilly W 1984 Rock and Mineral Magnetism (Glasgow: Blackie)

[34] Reufer M, Dietsch H, Gasser U, Hirt A, Menzel A and Schurtenberger P 2010 J. Phys. Chem. B 1144763

[35] Pike C R, Roberts A P and Verosub K L 1999 J. Appl. Phys. 85 6660-7

[36] Roberts A P, Liu Q, Rowan C J, Chang L, Carvallo C, Torrent J and Horng C-S 2006 J. Geophys. Res. $111 \mathrm{~B} 12 \mathrm{~S} 35$
[37] Stacey F D and Banerjee S K 1974 The Physical Principles of Rock Magnetism (Amsterdam: Elsevier)

[38] Dankers P 1981 Geophys. J. R. Astron. Soc. 64 447-61

[39] Pike C R, Roberts A P and Verosub K L 2001 Geophys. J. Int. $145721-30$

[40] Sacanna S, Rossi L and Philipse A P 2007 Langmuir 23 9974-82

[41] Frandsen C et al 2005 Phys. Rev. B 72214406

[42] Dunlop D J 1971 Ann. Geophys. 27 269-93

[43] De Boer C B, Mullender A T and Dekkers M J 2001 Geophys. J. Int. 146 201-16 